\section{Energy Focus}

Low bandgap, silole-containing heteroarene polymers developed for solar cells

The advantages of polymer solar cells (PSCs) for renewable energy include low cost, light weight, and ease of processing large surface areas. The highest power conversion efficiencies (PCEs) to date for PSCs have been achieved with bulk heterojunction devices composed of a polymer acting as electron donors and derivatized fullerenes as acceptors. Low-bandgap polymers are typically used to enhance light absorption, for example, benzodithiophene polymers, which achieve PCEs of over 7\%. Other polymer requisites are high hole (elec-

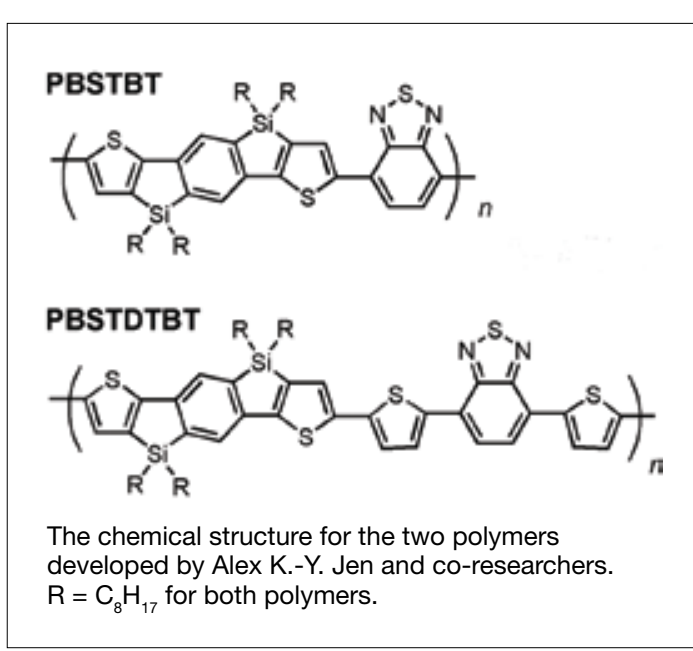

tron vacancy) mobility and the ability to form good films. Recently, A.K.-Y. Jen and co-researchers at the University of Washington, Seattle, designed and synthesized in high yield new low-bandgap copolymers that contain a thiophenephenylene-thiophene fused ring where silicon atoms replace the linked carbon atoms, resulting in low highest occupied molecular orbital (HOMO) levels and high hole mobilities.

As reported in the September 7th online edition of Chemistry of Materials (DOI:10.1021/cm1020228), Jen and co-researchers used the field-effect transistor (FET) technique to measure hole mobilities of PBSTBT and PBSTDTBT (see figure) to be $2.2 \times 10^{-3} \mathrm{~cm}^{2} \mathrm{~V}^{-1} \mathrm{~s}^{-1}$ and $1.1 \times 10^{-2} \mathrm{~cm}^{2} \mathrm{~V}^{-1} \mathrm{~s}^{-1}$, respectively.

Both polymers show good solubility in common organic solvents, an advantage in their device processing, and high thermal stability, with no transitions between $20^{\circ} \mathrm{C}$ and $350^{\circ} \mathrm{C}$ detected by differential scanning calorimetry.

Both polymers show long-wavelength absorption, which the researchers attribute to charge transfer from the benzobis (silolothiophene) donor to the acceptor elsewhere in the polymer chain. PBSTDTBT's absorption is much broader and at longer wavelengths than PBSTBT's, which the researchers said is due to the presence of two thiophene units between the donor and the acceptor compared to PBSTBT.

The researchers performed cyclic voltametry (CV) and density functional theory (DFT) calculations to determine HOMO and LUMO (lowest unoccupied molecular orbital) energy levels. Although DFT overestimated the orbital energy levels, it was consistent with the $\mathrm{CV}$ trends, for example, that the HOMO for PBSTBT lies lower in energy than PBSTDTBT's HOMO.

In addition, DFT predicts a lower lying HOMO for both polymers relative to their analogues where carbon replaces silicon, which is consistent with the researchers' desire to increase the polymers' open-circuit voltage.

The researchers fabricated PSCs with each polymer and determined good photo conversion efficiency from $400 \mathrm{~nm}$ to $700 \mathrm{~nm}$, with external quantum efficiencies about 50\% and PCEs about 3.5\%.

The researchers said that their work "provides a new method for synthesizing silole-containing heteroarenes, which show great potential in organic electronics applications, such as FETs and PSCs."

Steven Trohalaki

\section{Nano Focus}

Low-temperature aqueous synthesis yields large surface area tin oxide nanocrystals

$\mathrm{T}$ in oxide is a critical component of optical devices, lithium batteries, and conducting coatings, and used as a catalyst besides many other applications. Development of tin oxide particles with high surface area is particularly critical for use in chemical sensors and dyesensitive solar cells. Researchers from the National Institute of Advanced Industrial Science and Technology (AIST) in Japan have prepared tin oxide nanocrystals with unprecedented surface area using novel nanofabrication techniques.

As reported in the August issue of the Journal of the American Ceramic Society (DOI: 10.1111/j.15512916.201 0.03680.x; p. 2140), Y. Masuda and co-workers prepared nanocrystals by crystallization in the aqueous phase with minimal application of heat. Previous methods for fabricating particles have typically involved hightemperature annealing that causes aggregation and deformation resulting in reduced specific

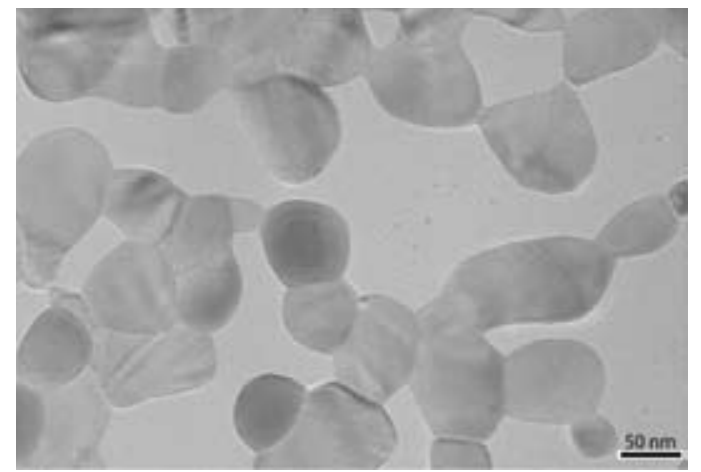

Transmission electron micrographs of tin oxide nanosheets prepared by precipitation from

low-temperature aqueous solution. Reproduced with permission from J. Am. Ceram. Soc. 93 (8) (2010) DOI: 10.1111/j.1551-2916.2010.03680.x; p. 2140. (C) 2010 The American Ceramic Society. 
surface area.

In the two-step process developed in this study, tin fluoride was first dissolved in water at $50^{\circ} \mathrm{C}$ for $20 \mathrm{~min}$. and the solution was then left undisturbed at $28^{\circ} \mathrm{C}$ for three days. Particles precipitated and settled at the bottom, and were collected and dried at $60^{\circ} \mathrm{C}$ for $12 \mathrm{~h}$.

Structural characterization using $\mathrm{x}$ ray diffraction and transmission elec- tron microscopy revealed that the precipitates were nanosheets composed of a mixture of $\mathrm{SnO}_{2}$ main phase and $\mathrm{SnO}$ additional phase. BrunauerEmmett-Teller (BET) surface area measured from nitrogen isotherms revealed a value of $194 \mathrm{~m}^{2} / \mathrm{g}$, more than double the highest value reported in previous studies whereas pore sizes were measured at $1-2 \mathrm{~nm}$.
The nanocrystalline sheets with large specific surface area produced in this technique are expected to be better suited for use in sensors. The researchers believe that this new fabrication method provides additional benefits as an eco-friendly alternative with reduced production costs through lower energy consumption and $\mathrm{CO}_{2}$ emission.

Kaushik Chatterjee

\section{Energy Focus}

Modified SMP allows high resolution mapping of lithium-ion diffusion

$\mathrm{L}$ ithium-ion batteries are one of the $U_{\text {most ubiquitous power sources for }}$ portable electronics, but the design of new batteries is currently limited by a poor understanding of the nanoscale mechanisms of lithium ion transport and how such mechanisms are affected by microstructure and defects. In the August 29th online edition of Nature Materials (DOI: 10.1038/nnano.2010.174), N. Balke of Oak Ridge National Laboratory (ORNL), A.N. Morozovska of the National Academy of Science of Ukraine, D.W. Chung of Purdue University, and their colleagues report on a novel method of mapping ion diffusion in $\mathrm{LiCoO}_{2}$ battery electrodes.

Using a modified scanning probe microscope (SPM) tip, the researchers applied a periodic electric field to induce the motion of lithium ions. The flux of ions into or out of the electrode is ac- companied by local structural changes that are detected by the deflection of the same tip. A combination of high frequency imaging and low frequency spectroscopy allows mapping of local ionic dynamics with nanoscale precision. The researchers estimate that this method offers a strain detection limit 6-8 orders of magnitude better than classical electrochemical techniques.

The team first applied a constant voltage to the SPM tip, causing a change in lithium ion concentration and an associated strain of the electrode. They observed changes in the electrode's morphology after biasing, as well as diffusion of ions across (001) planes.

They then applied a high-frequency periodic voltage, inducing changes in the resonance frequency resulting from cathode structure changes. The team mapped variations in Young's modulus and related them to fluctuations in lithium diffusion and intercalation behavior.

In their final experiment they varied both biasing pulse frequency and amplitude, allowing them to measure ion diffusion across large time scales in battery-like conditions with $<100 \mathrm{~nm}$ spatial resolution.

The use of the band excitation technology previously developed at ORNL has allowed the team to separate the electrochemical strain signal from the variations in surface topography that plague conventional SPM measurements. Dynamic hysteresis loop mapping has also helped the team to probe changes in lithium ion dynamics across a sample's surface and enhance its response at grain boundaries.

According to the researchers, this is the first time electrochemical reactivity has been observed on the level of a single structural defect. The researchers said that this novel combination of temporal and spatial resolution will help them study the local factors controlling electrochemical reactions, ultimately permitting the design of more efficient and reliable batteries.

Steven Spurgeon

\section{MRS eNewsletters \& Alerts}

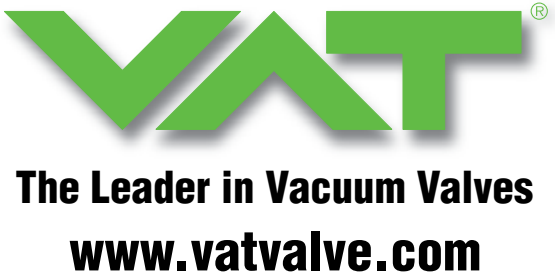

Vacuum Valves

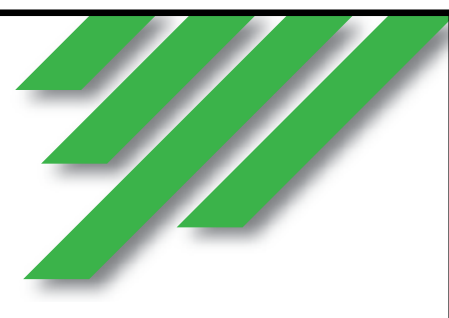

